\title{
ROCHE TOMOGRAPHY OF THE COOL STAR IN IP PEG
}

\author{
R. G. M. RUTTEN ${ }^{1,2}$, V. S. DHILLON ${ }^{2}$ \\ 1. Netherlands Foundation for Research in Astronomy \\ 2. Royal Greenwich Observatory, La Palma, Spain
}

\begin{abstract}
We analyse orbitally-resolved spectra of the $\mathrm{Na}$ I doublet in the dwarf nova IP Peg, using the newly developed technique of Roche tomography. We show that Roche tomography can be successfully employed to image the secondary stars in CVs, and that it also serves as an independent aid for determining binary system parameters. Our preliminary results suggest that the mass of the white-dwarf primary is $1.2 \mathrm{M}_{\odot}$, significantly higher than previous estimates.
\end{abstract}

\section{Introduction}

A complete understanding of CVs is impossible without understanding the cool secondary stars (typically $\mathrm{K}$ or $\mathrm{M}$ dwarfs). From studies of the secondary star we may obtain insight into, for example, the binary star parameters, magnetic activity on its surface (and thus on the evolution of CVs), on the mass-transfer process, and the disk's radiation field through the study of irradiation. This makes detailed study of the secondary stars worthwhile, although their faintness in comparison to the usually much brighter accretion disk renders them difficult objects to study.

IP Peg is a dwarf nova, and arguably one of the best studied cataclysmic variables (see Dhillon \& Rutten 1995 and reference therein). The secondary star in IP Peg has been detected through the presence of ellipsoidal variations (Martin, Jones \& Smith 1987). Also, spectral features in IP Peg, such as the $\mathrm{NaI}$ doublet at $\lambda \lambda 8183,8195 \AA$, are formed in the photosphere of the secondary star, as is shown by radial velocity studies (Martin et al. 1989). These lines can be used to study secondary star characteristics, and, when observed with sufficiently high resolution and as a function of orbital phase, can be used to map the surface of the secondary star, as we will show in this paper. The results presented here are of a preliminary nature; a full analysis will be published elsewhere. 


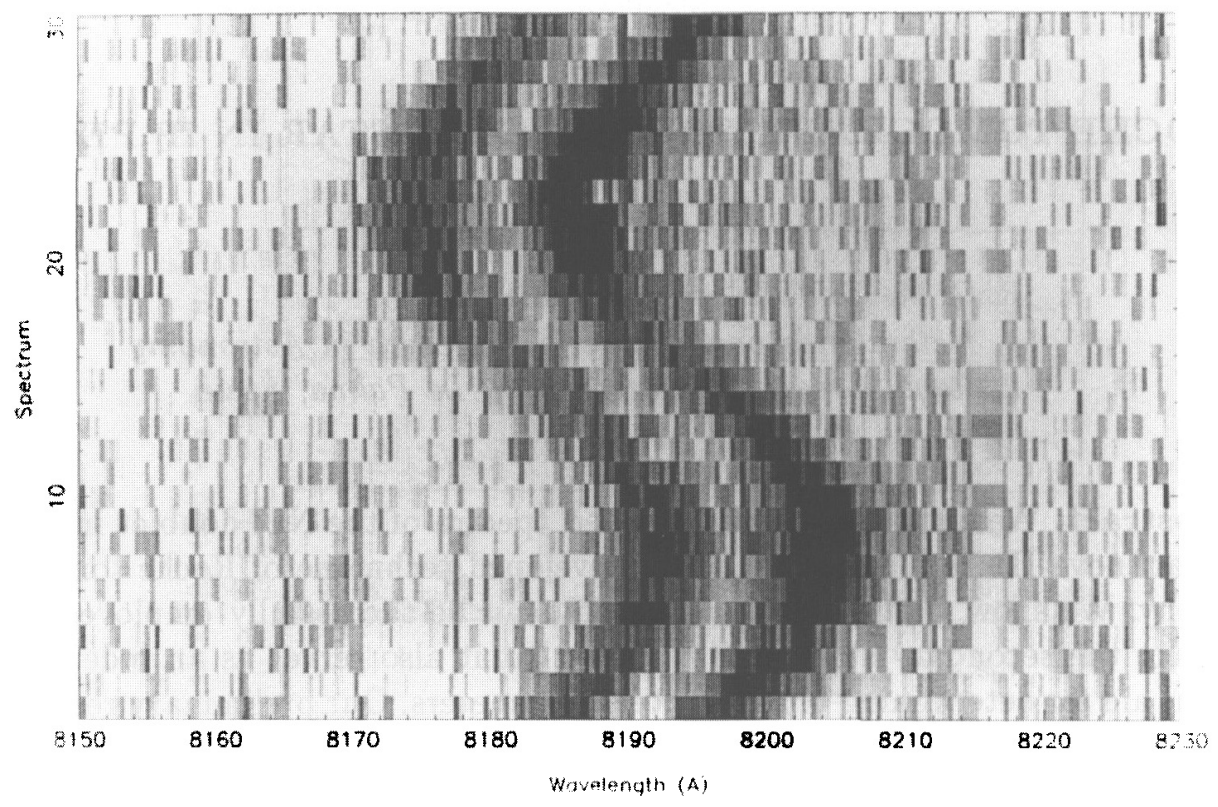

Figure 1. Trailed spectrum of the Na I doublet in IP Peg.

\section{Observations}

The phase-resolved spectra of the Na I doublet of IP Peg in quiescence were obtained on two nights in 1994 August with the ISIS dual-beam spectrograph at the Cassegrain focus of the $4.2 \mathrm{~m}$ William Herschel Telescope on the island of La Palma, Spain. The phase-folded spectra of the Na I doublet, averaged into 30 phase bins, are presented in Fig. 1 as a trailed spectrum. Clearly visible are the orbital radial velocity variations, and variations in line strength as well as line width.

\section{Roche tomography}

The observed line profile at a given orbital phase is the sum of line contributions from all visible parts of the star. The orbital motion of the star introduces Doppler shifts of the line features as a whole, and changes the line strength and width. The observed line profile will also depend on how the line strength varies over the stellar surface, and its detailed signature depends on the geometry of the binary. By modeling the observed line shape as a function of orbital phase, information may be deduced as to how the line strength varies over the stellar surface; this is Roche tomography (Rutten \& Dhillon 1994). 


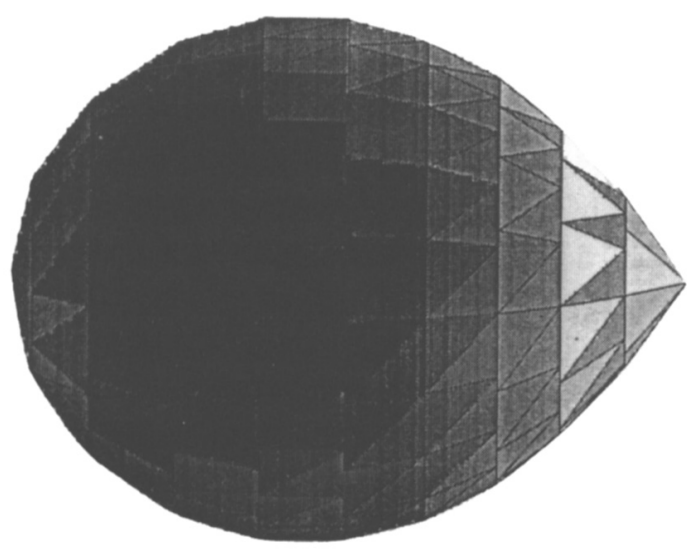

Figure 2. Representation of the optimum NaI brightness map on the surface of the secondary star in IP Peg. Dark areas indicate stronger Na I absorption.

In our implementation of Roche tomography the stellar surface is modelled by a large number of triangular tiles. The photosphere coincides with the critical Roche surface, and the star is locked into orbital motion. Furthermore, it is assumed that the line shape does not change over the stellar surface, but its strength may. It is this strength of the line for each surface element which is adjusted when fitting the data. Optimization of the fit is obtained using the maximum-entropy criterion, which arrives at the smoothest possible solution which fits the data to within the errors.

\section{Analysis and results}

The brightness map of the $\mathrm{Na}$ I doublet on the surface of the secondary star of IP Peg, computed from the data in Fig. 1, is shown in Fig. 2. This map shows that the $\mathrm{NaI}$ line is less strong on the hemisphere facing the white dwarf. Presumably heating by irradiation causes a reduced line strength.

So far, we have assumed that the binary star geometry is known. However, published binary star parameters are not in agreement over what the masses of the components are, which renders the results from Roche tomography less certain. To overcome this problem, rather than picking just one set of binary star masses, we reconstruct the Na I brightness map of the secondary star using a large number of combinations of masses, and from the set of solutions choose the one which, in line with the maximum entropy criterion, has the least structure. This method basically extends the entropy optimization by including the masses as additional free param- 


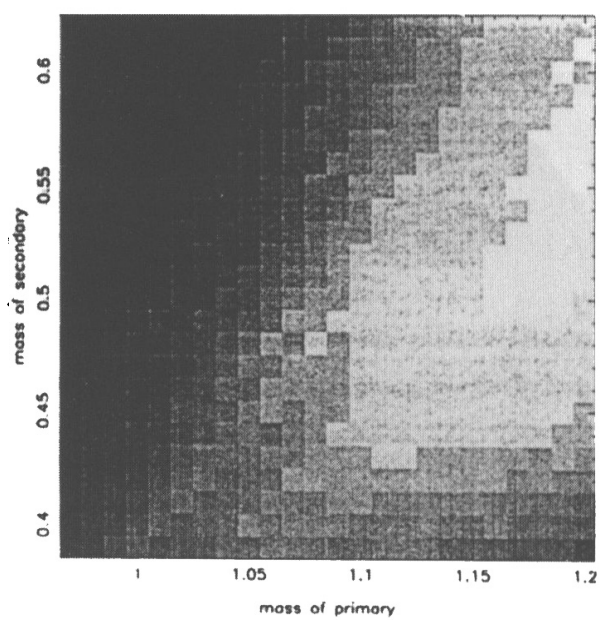

Figure 3. Entropy landscape for IP Peg where the smoothness parameter (entropy) of the fit is plotted against the masses of the binary star components. The brightest point indicates the smoothest map.

eters. Fig. 3 shows the so-called entropy landscape, which plots entropy as a function of masses of the primary and secondary star (see examples in Rutten \& Dhillon 1994). Our preliminary results suggest the optimum fit is reached for $M_{1}=1.2 \mathrm{M}_{\odot}$ and $M_{2}=0.55 \mathrm{M}_{\odot}$. The radial velocities corresponding to this solution agree well with those measured from our data, as well as with published radial velocities. Note that Roche tomography automatically takes irradiation effects into account, and hence no $a$ posteriori corrections are needed as in the case of masses determined from radial velocities only. Hence a mass determination from Roche tomography is potentially more reliable.

\section{Conclusions}

It is shown that Roche tomography is capable of producing surface maps of the secondary stars in cataclysmic variables. The same technique also results in a new and independent method for estimating binary star parameters, the strength of which is that it does not just attempt to fit one aspect of the line profile, e.g. the radial velocity, but rather fits the whole line profile and its variations as a function of orbital phase.

\section{References}

Dhillon, V.S., Rutten, R.G.M., 1995, MNRAS, 274, 27

Martin, J.S., Friend, M.T., Smith, R.C., Jones, D.H.P., 1989, MNRAS, 240, 519

Martin, J.S., Jones, D.H.P., Smith, R.C., 1987, MNRAS, 224, 1031

Rutten, R.G.M., Dhillon, V.S., 1994, A\&A, 288, 773 